\title{
Study of Atomically Controlled Au Nanoclusters Using Aberration-Corrected STEM
}

\author{
N. Bhattarai, D. Bahena, U. Santiago, A. Tlahuice, A. Ponce, and M. Jose-Yacaman \\ Department of Physics and Astronomy, University of Texas at San Antonio, One UTSA Circle, San \\ Antonio, TX , 78249 USA
}

The total determination of structure of molecular nanocrystal by X-ray diffraction has been achieved only in few cases as it requires homogenous, high degree of ordering, single crystal of oriented molecules[1,2]. Presented here is an alternative technique that can be used in full determination of molecular crystal structures of nanoclusters $\left(\mathrm{Au}_{144}\right.$ and $\left.\mathrm{Au}_{333}\right)$ without the fabrication of single crystal. We fabricate those nanoclusters by using modified Brust's method and analyze using UV/Visible spectrum and MALDI TOF mass spectrometry[3]. We propose the combination of low voltage scanning transmission electron microscopy (STEM) electron diffraction and high angle annular dark field (HAADF) STEM images in a Cs-corrected JEOLJEM-ARM STEM to determine the crystal structure. The electron diffraction patterns and HAADF-STEM images are compared with the theoretical simulations of images and diffraction patterns obtained from atomistic structural models derived through first-principles density functional theory (DFT) calculations.

It should be noted that the cleanliness of the sample, operating voltage of the microscope and exposure time of beam to the sample plays significant role in order to get atomically resolved images without altering the particle shape and attached ligands. The HAADF STEM images obtained for $\mathrm{Au}_{144}$ nanocluster is presented in Fig. 1a-d and the corresponding FFT pattern is inset in each case. Based on the number of reflections and angles from FFT pattern, the corresponding structure is simulated. Fig. 2a-d represents the atomically resolved HAADF STEM images obtained for $\mathrm{Au}_{333}$ nanoclusters and the corresponding FFT pattern is inset on it. In most of the cases, 9 atomic layers are clearly visible. The STEM electron diffraction of $\mathrm{Au}_{144}$ and $\mathrm{Au}_{333}$ nanocluster is presented in Fig. $3 \mathrm{a}$ and $\mathrm{b}$ respectively. The number of reflection points and angle between them is measured and compared with the simulated ones. The combination of HAADF-STEM images (experimental and simulated) with diffraction patterns (experimental and simulated) will be used to investigate the crystal structure.

\section{References:}

[1]Tran, N. T.; Powell, D. R.; Dahl, L. F. Angewandte Chemie International Edition 2000, 39, 4121.

[2]Whetten, R. L.; Price, R. C. Science 2007, 318, 407.

[3]Brust, M.; Schiffrin, D. J.; Bethell, D.; Kiely, C. J., Advanced materials 1995, 7, 795.

[4]Acknowledgments: The authors would like to acknowledge NSF grants DMR-1103730, "Alloys at the Nanoscale: The Case of Nanoparticles Second Phase" and PREM: NSF PREM Grant \# DMR 0934218. 

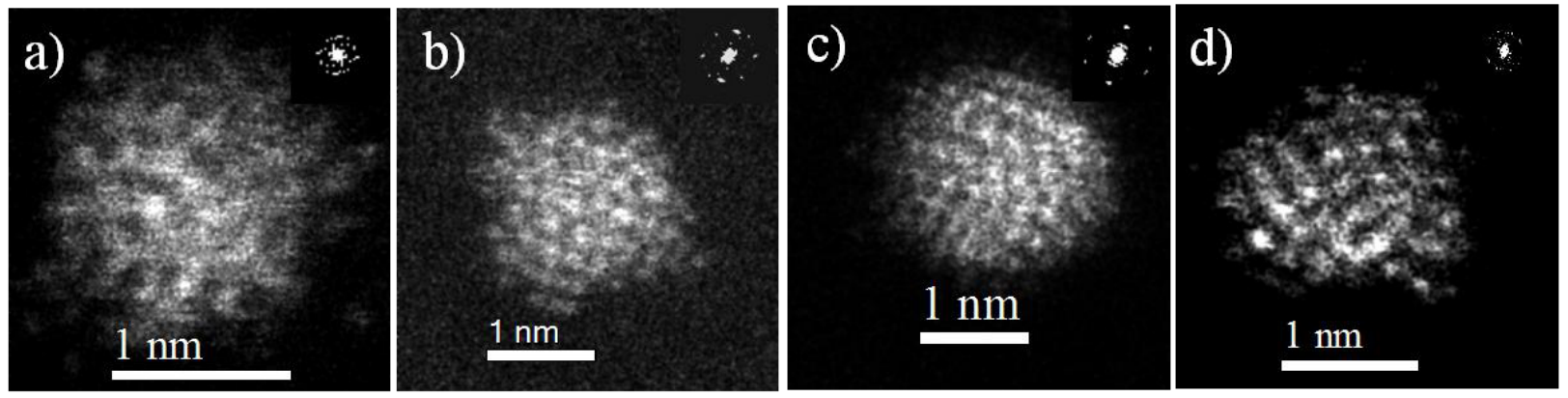

Fig. 1. a)-d) HAADF STEM images of $\mathrm{Au}_{144}$ nanocluster oriented along different directions. The corresponding FFT pattern is inset in STEM image.
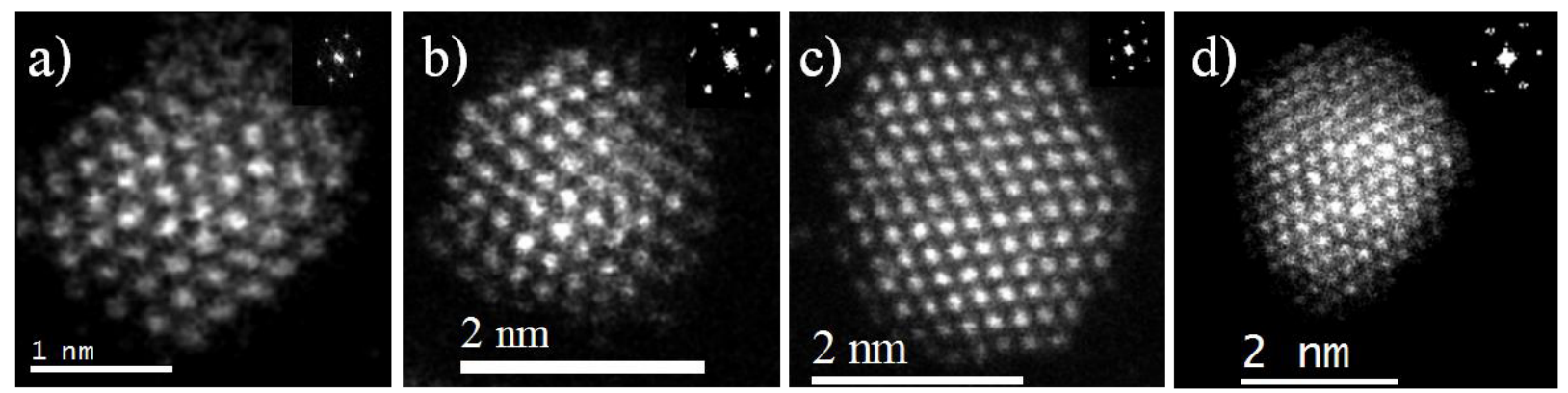

Fig. 2. a)-d) Some representative HAADF STEM images of $A_{333}$ nanocluster along different orientations. The corresponding FFT pattern is inset in each image.
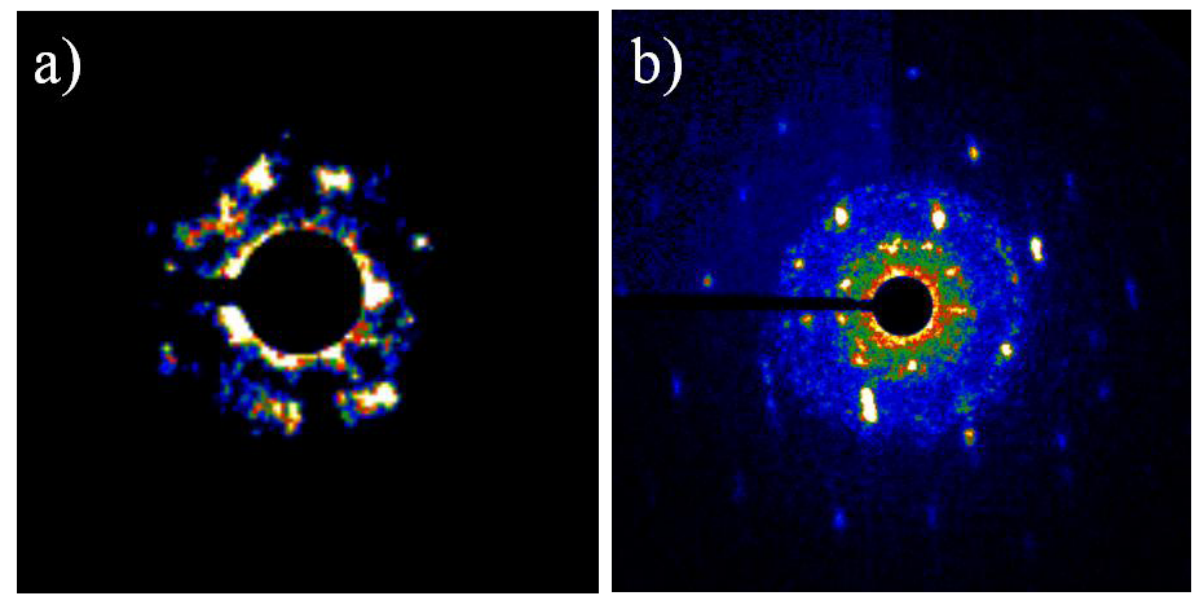

Fig. 3. STEM diffraction patterns from $\mathrm{Au}_{144}(\mathrm{a})$ and $\mathrm{Au}_{333}$ (b) nanocluster. The obtained diffraction pattern is compared with the diffraction patterns from atomistic structural models. 